\title{
Low threshold linear cavity mode-locked fiber laser using microfiber-based carbon nanotube saturable absorber
}

\begin{abstract}
ABSTRCT
In this work, we demonstrate a linear cavity mode-locked erbium-doped fiber laser in C-band wavelength region. The passive mode-locking is achieved using a microfiber-based carbon nanotube saturable absorber. The carbon nanotube saturable absorber has low saturation fluence of $0.98 \mu \mathrm{J} / \mathrm{cm} 2$. Together with the linear cavity architecture, the fiber laser starts to produce soliton pulses at low pump power of $22.6 \mathrm{~mW}$. The proposed fiber laser generates fundamental soliton pulses with a center wavelength, pulse width, and repetition rate of $1557.1 \mathrm{~nm}, 820 \mathrm{fs}$, and $5.41 \mathrm{MHz}$, respectively. This mode-locked laser scheme presents a viable option in the development of low threshold ultrashort pulse system for deployment as a seed laser.
\end{abstract}

Keyword: Ultrashort pulse; Pulse fiber laser; Carbon nanotube. 